\title{
Modeling evolution of composition patterns in a binary surface alloy
}

\author{
Mikhail Khenner*1,2 and Victor Henner ${ }^{3,4,5}$ \\ ${ }^{1}$ Department of Mathematics, Western Kentucky University, Bowling Green, KY 42101, USA \\ ${ }^{2}$ Applied Physics Institute, Western Kentucky University, Bowling Green, KY 42101, USA \\ ${ }^{3}$ Department of Theoretical Physics, Perm State University, Perm, 614990 Russia \\ ${ }^{4}$ Department of Mathematics, Perm National Research Polytechnic University, Perm, 614990, Russia \\ ${ }^{5}$ Department of Physics, University of Louisville, Louisville, KY 40222, USA
}

(Dated: October 15, 2020)

\begin{abstract}
Evolution of composition patterns in the annealed, single-crystal surface alloy film is considered in the presence of the spinodal decomposition, the compositional stress and the diffusion anisotropy. While the former two effects contribute to overall phase separation, the anisotropy, correlated with the surface crystallographic orientation, guides the in-plane formation and orientation of a pattern. The impacts of the anisotropy parameters on patterns are systematically computed for [110], [100], and [111]-oriented fcc cubic alloy surfaces.
\end{abstract}

\section{INTRODUCTION}

Surface alloying is usually defined as the process whereby the atoms of metal B, adsorbed on a surface of metal A, intermix with that surface. The metals may or may not be miscible in the bulk phase 1, 2. Surface alloying often occurs in the initial stages of a heteroepitaxial metal-on-metal growth. Once mixed into a surface layer, B atoms may form patterns due to thermodynamic or kinetic instabilities. Formation of such surface composition patterns is important for heterogeneous catalysis, corrosion, lubrication and adhesion. Also the electric, magnetic, plasmonic, and photovoltaic properties of a surface can be strongly influenced by the composition of the near surface region.

Surface alloying is prominent in the copper-based ( $\mathrm{CuPd}, \mathrm{CuAu}, \mathrm{CuNi}, \mathrm{CuPt}, \mathrm{CuAg})$ and nickel-based (NiPd, $\mathrm{NiCu}, \mathrm{NiAu}, \mathrm{NiPt}$ ) systems [1. Here the first metal is the substrate and the second metal is the overlayer. A semiconductor-on-metal systems that result in a surface alloy or a surface phase separated states also have been studied 3 [5].

Significant experimental efforts were aimed at understanding the conditions that result in surface alloying or dealloying (i.e., phase separation [6]) in various metal systems as well as at characterization of the surface composition patterns [1, 2, 7-9]. There is understanding that kinetic factors strongly impact surface alloy formation and therefore they also should play a significant role in the evolution of the composition of the surface upon annealing. For instance, in Table 3 Besenbacher et al. 1] summarize the available information on a surface alloy formation for various permutations of six metals: $\mathrm{Ni}, \mathrm{Pd}, \mathrm{Pt}, \mathrm{Cu}, \mathrm{Ag}$ and $\mathrm{Au}$, including three low-index surfaces for each substrate metal. This gives the total of 108 combinations, and in 36 cases (30\%) it is not $100 \%$ certain whether the surface alloy forms or not. The single stated reason for the uncertainty is the kinetic effects that "may hinder the system from reaching the thermodynamic stable configuration, such as formation of a surface alloy. ...". Despite this admitted importance of the kinetic factors they, to our knowledge, were not specifically targeted for experimental studies, and they are infrequently remarked upon in the literature. In fact, we found only one explicit mention in Ref. 8], where the coverage-dependent mobility of $\mathrm{Bi}$ atoms is cited as the cause of an unusual formation of surface alloy domains.

Tersoff [10] explained surface alloy formation using the energetic model that accounts only for compositional strain (i.e., strain that emerges due to the size mismatch of A and B atoms), and ignoring any kinetic effects. He also presented a few results of a small-scale Monte Carlo simulations of the distribution of $\mathrm{Au}$ atoms on $\mathrm{Ni}(001)$. For sub-monolayer equilibrium Au coverage at $600 \mathrm{~K}$, Au atoms intermix with the first four atomic layers on Ni surface, and $\mathrm{Au}$ concentration decreases linearly with the atomic layer number. Upon annealing at very low coverage, the composition domains are formed in the first layer. They show the tendency toward clustering with increasing interface energy $\gamma_{A B}$, corresponding to a compositional strain energy. This energy is introduced in ad hoc fashion simply by adding a fixed energy $\Delta_{A B}$ for each bond between an $\mathrm{A}$ atom and $\mathrm{B}$ atom. To our knowledge there is no published continuum model specifically for surface alloying.

In this paper we construct a basic continuum multiple-parameter model for the dynamics of a surface composition in the presence of the spinodal decomposition [11, 12, the compositional strain, and the diffusional anisotropy. This anisotropy is the important kinetic factor in, for example, meandering of steps on vicinal surfaces 13, control of surface nanostructure by electromigration [14, 15, formation of heteroepitaxial quantum dots [16, and ion-beam

* Corresponding author. E-mail: mikhail.khenner@wku.edu. 
sputtering [17. Using the model, we perform the computational study of the self-assembled composition patterns on three major low-index crystal surfaces. Also we show and discuss how the pattern characteristic features vary when the dimensionless parameters governing the strengths of the above-listed physical effects are varied. Impacts of the electromigration on the formation and evolution of composition patterns are studied in the recent paper by one of the authors [18].

\section{MODEL FORMULATION}

The physical scenario for our model is as follows. Initially, a planar film (the substrate) is composed of A atoms. B atoms are deposited onto the substrate surface and adsorb there, thus forming a surface mixture of $\mathrm{A}$ and $\mathrm{B}$ atoms (a substitutional $\mathrm{A}_{1-x} \mathrm{~B}_{x}$ surface alloy, see Fig. 1). Once a pre-set concentration of B atoms in the surface mixture has been reached, the flux of $\mathrm{B}$ atoms is turned off and the film enters the annealing stage. This activates the diffusion in the surface mixture and results in the evolution of the surface composition. This annealing stage is the initial condition for the model. The diffusion of B out of the mixed surface layer and into the substrate is assumed negligible on the time scale of the compositional pattern formation in the surface layer, which is estimated on the order of minutes, see Sec. III. This assumption is justified, since in alloy systems with substantial mismatch in atomic sizes the compositional strain energy is larger in the bulk than at the surface by at least one order of magnitude. As a result, B atoms strongly prefer to be at the surface, even in the absence of any surface energy effects [10]. 29]

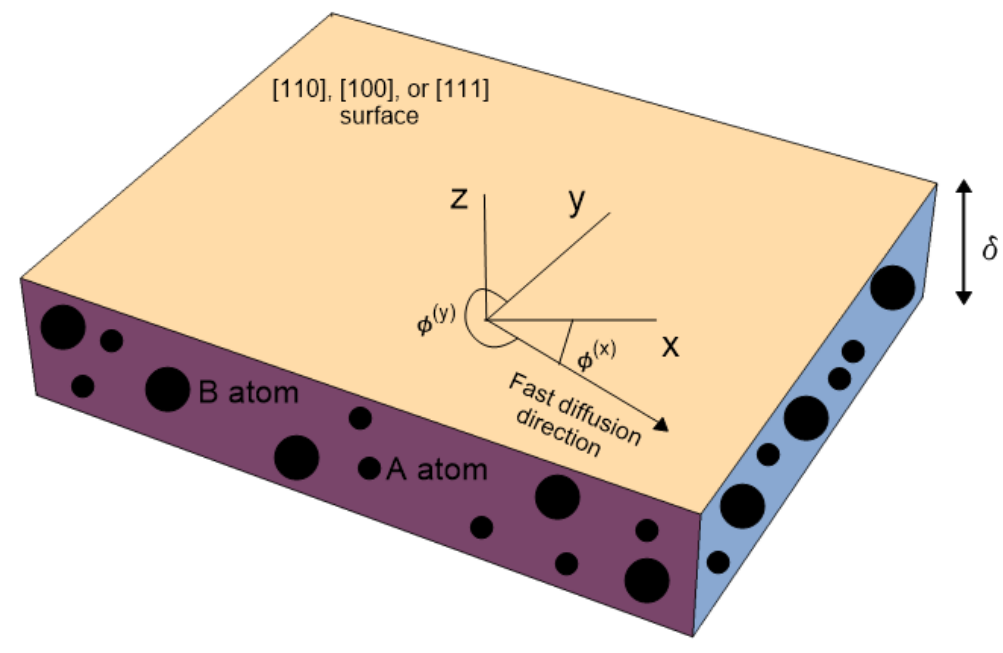

FIG. 1: Schematic representation of a surface alloy. $x$ and $y$ axes of the Cartesian reference frame are in the film/substrate plane. The $z$ axis is perpendicular to that plane, and it points into the vapor or vacuum above the film.

Let $\nu_{A}$ and $\nu_{B}$ be the number of atoms of the components A and B per unit surface area after formation of a mixed surface layer. Then the dimensionless surface concentrations, or the local composition fractions, are defined as $C_{i}=\nu_{i} / \nu, \nu=\nu_{A}+\nu_{B}$, with

$$
C_{A}(x, y, t)+C_{B}(x, y, t)=1
$$

due to the negligible vacancy concentration. $C_{i}(x, y, t)$ is in fact the average of a $3 \mathrm{D}$ concentration profile over the surface layer thickness $\delta, C_{i}(x, y, t)=(1 / \delta) \int_{0}^{\delta} c_{i}(x, y, z, t) d z$. $\delta$ typically does not exceed five atoms [10, and we take it equal to the size of two B atoms. [30. We proceed to construct a diffusion model that enables to follow the spatio-temporal evolution of $C_{B}$. Notice that this is sufficient, since due to Eq. (1) the evolution of $C_{A}$ follows trivially from equation $\partial C_{A} / \partial t=-\partial C_{B} / \partial t$ (thus if $C_{B}$ increases locally, i.e. at some point $(x, y)$ in the film, then $C_{A}$ decreases at that point with the same rate).

The diffusion equation reads

$$
\frac{\partial C_{B}}{\partial t}=-\frac{\Omega}{\delta} \nabla \cdot \boldsymbol{J}_{B}
$$

where $\Omega$ is the atomic volume and $\boldsymbol{\nabla}=\left(\partial_{x}, \partial_{y}\right)$. Since the mass transport is driven by the gradient of the chemical potential $\mu_{B}$, the flux $\boldsymbol{J}_{B}$ is given by

$$
\boldsymbol{J}_{B}=-\frac{\nu_{B}}{k T} \boldsymbol{D}_{B} \cdot \nabla \mu_{B}=-\frac{\nu}{k T} C_{B} \boldsymbol{D}_{B} \cdot \nabla \mu_{B},
$$


where $\boldsymbol{D}_{B}$ is the diffusion tensor and $k T$ the Boltzmann's factor.

The chemical potential in Eq. (3) is the sum of the contributions due to alloy thermodynamics and the compositional stress [19]21]:

$$
\mu_{B}=\Omega\left[\frac{1-C_{B}}{\delta} \frac{\partial \gamma}{\partial C_{B}}+\tau \eta_{0}\left(1-C_{B}\right) C_{B}-\epsilon \nabla^{2} C_{B}\right]
$$

where $\gamma$ is the free energy, $\epsilon$ the Cahn-Hilliard gradient energy coefficient, $\eta_{0}>0$ the effective solute expansion coefficient, and $\tau= \pm 1$. The compositional stress term $\tau \eta_{0}\left(1-C_{B}\right) C_{B}$ is stated in the first order of the perturbation theory [21, which assumes that in the zeroth order the compositional stress is zero (the reference base state of stress). The effective solute expansion coefficient measures the relative size difference of A and B atoms and quantifies the linear lattice strain due to increasing the concentration of B. $\eta_{0}>0$ corresponds to large B atoms. With this choice, the choice of $\tau=1$ or -1 depends on whether the stress is tensile or compressive. According to Ref. 21 replacing a larger B atoms with a smaller A atoms increases the energy if the stress is tensile. This implies the destabilization of the base uniform composition state in the linear stability analysis (LSA) (and facilitation of phase separation in the nonlinear regime). Thus we select $\tau=-1$, since the negative value provides the destabilization effect at the considered average surface composition, see LSA at the end of this section.

In Eq. 4

$$
\gamma=\gamma_{A}\left(1-C_{B}\right)+\gamma_{B} C_{B}+k T \nu\left[\left(1-C_{B}\right) \ln \left(1-C_{B}\right)+C_{B} \ln C_{B}+H\left(1-C_{B}\right) C_{B}\right]
$$

is the total free energy 20, 22. Here $\gamma_{A}$ and $\gamma_{B}$ are the surface energies of the alloy components, $k T \nu$ is the alloy entropy, and the dimensionless number $H=\alpha_{i n t} / k T \nu$ measures the bond strength relative to the thermal energy $k T$. Here $\alpha_{i n t}$ is the enthalpy. The first two terms are the weighted surface energy, and the last two terms constitute the regular solution model.

Finally, the diffusivity, $\mathbf{D}_{B}$, is given by a transversely isotropic diffusion tensor

$$
\mathbf{D}_{B}=\left(\begin{array}{cc}
D_{B}^{(x x)} & 0 \\
0 & D_{B}^{(y y)}
\end{array}\right)=\left(\begin{array}{cc}
D_{B, \min }^{(x x)} f\left(\phi^{(x)}\right) & 0 \\
0 & D_{B, \min }^{(y y)} f\left(\phi^{(y)}\right)
\end{array}\right)
$$

where $f\left(\phi^{(x)}\right)=1+\beta \cos ^{2}\left[m \phi^{(x)}\right], f\left(\phi^{(y)}\right)=1+\beta \cos ^{2}\left[m \phi^{(y)}\right]$ are the surface diffusional anisotropy functions for facecentered cubic (fcc) crystals [14, 16]. $\beta \geq 0$ is the strength of crystallographic anisotropy and $\phi^{(\alpha)}(\alpha=x, y)$ are the misorientation angles formed between the $\alpha$-axis and the fast surface diffusion direction. Note that $f\left(\phi^{(x)}\right), f\left(\phi^{(y)}\right) \geq 1$ (are positive). The integer $m=1,2,3$ is determined by the crystallographic orientation of the film surface. For $m=1$ ([110] surface): $\phi^{(y)}=\pi / 2+\phi^{(x)}, 0 \leq \phi^{(x)} \leq \pi / 2$; for $m=2\left([100]\right.$ surface): $\phi^{(y)}=\phi^{(x)}, 0 \leq \phi^{(x)} \leq \pi / 4$; for $m=3$ ([111] surface): $\phi^{(y)}=\pi / 6+\phi^{(x)}, 0 \leq \phi^{(x)} \leq \pi / 6[14]$.

Choosing the typical thickness $h$ of the as-deposited surface alloy film as the length scale, $k T h^{2} \delta^{2} / \Omega^{2} \nu D_{B, m i n}^{(x x)} \gamma_{B}$ as the time scale, and combining equations yields the dimensionless $\mathrm{PDE}$ for $C_{B}(x, y, t)$ :

$$
\frac{\partial C_{B}}{\partial t}=\boldsymbol{\nabla} \cdot\left[C_{B} \nabla_{\Lambda B}\left(\left(1-C_{B}\right) \frac{\partial \gamma}{\partial C_{B}}-G^{(C H)} \nabla^{2} C_{B}+\tau S C_{B}\left(1-C_{B}\right)\right)\right]
$$

where

$$
\gamma=\Gamma\left(1-C_{B}\right)+C_{B}+N\left[\left(1-C_{B}\right) \ln \left(1-C_{B}\right)+C_{B} \ln C_{B}+H\left(1-C_{B}\right) C_{B}\right]
$$

The dimensionless parameters are the ratio of the pure energies $\Gamma=\gamma_{A} / \gamma_{B}$, the entropy $N=k T \nu / \gamma_{B}$, the enthalpy $H=\alpha_{i n t} / k T \nu$, the Cahn-Hilliard gradient energy coefficient $G^{(C H)}=\epsilon \delta / \gamma_{B} h^{2}$, and the solute expansion coefficient $S=\eta_{0} \delta / \gamma_{B}$. Also $\nabla_{\Lambda B}=\left(f\left(\phi^{(x)}\right) \partial_{x}, \Lambda_{B} f\left(\phi^{(y)}\right) \partial_{y}\right)$ is the anisotropic gradient operator, where $\Lambda_{B}=D_{B, m i n}^{(y y)} / D_{B, m i n}^{(x x)}$ is the dimensionless ratio of the amplitudes of the diagonal components of the anisotropic diffusivity tensor. We will call $\Lambda_{B}$ the diffusional anisotropy. At $\Lambda_{B}=1$ the diffusional anisotropy is zero. (Of course, the separation into crystallographic and diffusional anisotropy is only for convenience of discussion. Both anisotropies stem from the anisotropy of diffusion in the surface layer of a single-crystal film, see Eq. (6).)

Remark 1. Setting $\beta=0$ amounts to consideration of a generic film surface, since in this case the crystallographic orientation parameter $m$ is irrelevant. $\phi^{(x)}$ is also irrelevant, and $f\left(\phi^{(x)}\right)=f\left(\phi^{(y)}\right)=1, \nabla_{\Lambda B}=\left(\partial_{x}, \Lambda_{B} \partial_{y}\right)$. It follows that if $\Lambda_{B}=1$ in addition to $\beta=0$, then the diffusion tensor is isotropic, with identical diagonal components $\left(\mathbf{D}_{B}=D_{B} \boldsymbol{I}\right.$, where $D_{B}$ is the diffusivity and $\boldsymbol{I}$ the identity tensor $)$, and also $\boldsymbol{\nabla}_{\Lambda B}=\boldsymbol{\nabla}$. In this case the diffusion in the film is isotropic. 
Remark 2. When the crystallographic anisotropy strength $\beta>0$, the anisotropic gradient operator $\nabla_{\Lambda B} \neq \boldsymbol{\nabla}$ even at zero diffusional anisotropy, due to intrinsic reconstruction of a particular low-index crystal surface. The overall anisotropy effect is enhanced if in addition to a non-zero crystallographic anisotropy the diffusional anisotropy is also present.

The anisotropy functions for the planar surface, $f\left(\phi^{(x)}\right)$ and $f\left(\phi^{(y)}\right)$ are plotted in Fig. 2 , At $\beta$ and $m$ fixed, since $\phi^{(y)}=\phi^{(y)}\left(\phi^{(x)}\right)$, these functions depend on $\phi^{(x)}$ only. It can be seen that for $m=1$ and $m=3$ the shape of these functions is the same on their domains. Thus one should anticipate the identical computational results for $m=1$ and $m=3$ when $\phi^{(x)}$ is chosen symmetrically with respect to the center of the admissible interval, $0 \leq \phi^{(x)} \leq \pi / 2$ for $m=1$, or $0 \leq \phi^{(x)} \leq \pi / 6$ for $m=3$. In other words, it is expected that a composition pattern obtained at $m=1$ is also the pattern at $m=3$, but at a different value of $\phi^{(x)}$, which can be obtained by a simple linear map of one interval onto another.

(a)

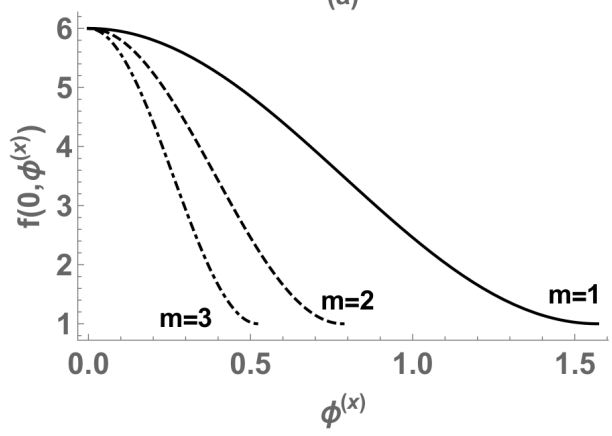

(b)

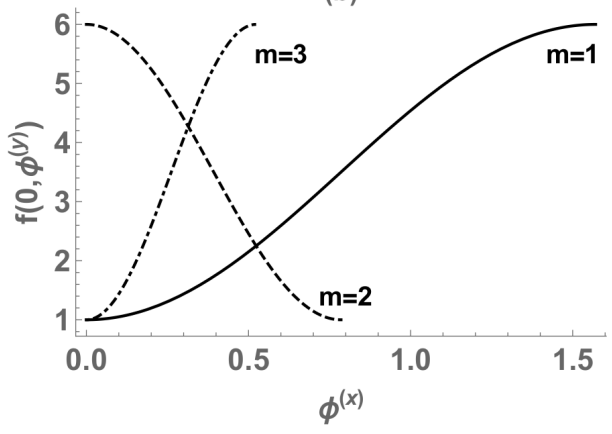

FIG. 2: The anisotropy functions at $m=1,2,3$ and $\beta=5$.

In this paper, the thermodynamic parameters $\Gamma, H$, and $N$ in Eq. (8) will be permanently chosen such that the free energy $\gamma\left(C_{B}\right)$ is a double-well curve shown in Fig. 3. In this case Eq. (7) forces a thermodynamic phase separation (spinodal decomposition) of the surface layer, which in the absence of other factors (stress, anisotropy, etc.) favors two stable composition states, $A_{0.85} B_{0.15}$ and $A_{0.15} B_{0.85}$. (It can be easily shown that $\gamma$-curve is convex when $H \leq 2$, thus spinodal decomposition emerges at $H>2$, or $T<T_{c}=\alpha_{\text {int }} / 2 k \nu\left[22\right.$. For the parameters in Table I] $T_{c}=1163 \mathrm{~K}$.) In Appendix we further comment on thermodynamic aspects of the model.
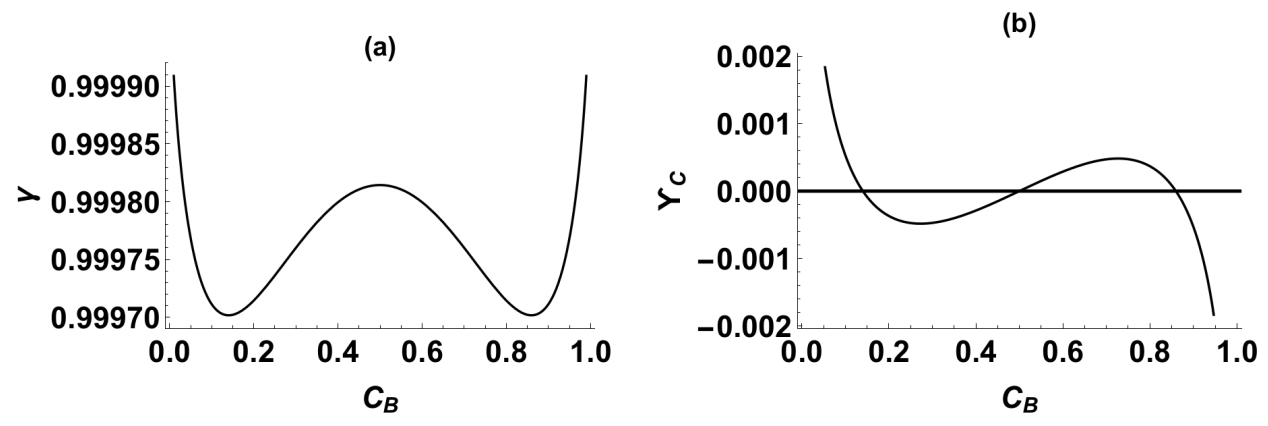

FIG. 3: (a) The dimensionless free energy $\gamma$, and (b) $\Upsilon_{C} \equiv-\partial \gamma / \partial C_{B}$ for a binary system with a miscibility gap. $\Gamma=1$, $H=2.515, N=0.0029, G^{(C H)}=1.5 \times 10^{-5}$. (These dimensionless values correspond to the physical values in Table $\mathrm{I}$ )

For computations of Eq. (7) we employ in Sec. III the method of lines framework with a pseudospectral spatial discretization on 140x140 rectangular grid and a stiff ODE solver in time. Periodic conditions are imposed on the boundary of a square computational domain with the dimensions $\left[7 \lambda_{\max }, 7 \lambda_{\max }\right]$. According to LSA, here $\lambda_{\max }$ is the wavelength of the fastest growing unstable mode of the uniform solution $C_{B}=C_{B 0}$. The LSA of Eq. 7 yields the perturbation growth rate

$$
\omega\left(k_{1}, k_{2}\right)=C_{B 0}\left[k_{1}^{2} W-G^{(C H)}\left(k_{1}^{4}+k_{1}^{2} k_{2}^{2}\right)\right] f\left(\phi^{(x)}\right)+C_{B 0} \Lambda_{B}\left[k_{2}^{2} W-G^{(C H)}\left(k_{2}^{4}+k_{1}^{2} k_{2}^{2}\right)\right] f\left(\phi^{(y)}\right)
$$

with

$$
W=1-\Gamma-\tau S\left(1-2 C_{B 0}\right)+H\left(3-4 C_{B 0}\right)+N \frac{C_{B 0} \ln \left[C_{B 0} /\left(1-C_{B 0}\right)\right]-1}{C_{B 0}}
$$


and where $k_{1}$ and $k_{2}$ are the perturbation wavenumbers in the $x$ and $y$ directions. $\omega$ is plotted in Fig. 4 at $C_{B 0}=0.3$ and $\tau=-1$ (the choice for the computation in Sec. III). The linear instability has a long-wavelength character, and the compositional stress shifts the maximum of $\omega$ towards small wavelengths. These results point to the continuous coarsening of a non-uniform initial composition field, i.e. the formation of the surface domains with the ever increasing size by merging of a smaller domains; notice that the domain coarsening may run in parallel to overall phase separation within the surface layer. Such behavior is indeed seen in the computations discussed in the next two sections. The computations show the development of the late stages in the coarsening process of the composition domains. It is appropriate to remark here that our model presently does not incorporate a phase refining mechanism, such as the stress in the bulk layers of the film. Such stress may have the impact on the surface layer and result in interrupted coarsening and thus in composition domains of a certain fixed size [22. $k_{\max }=8$ is chosen for all simulations, which corresponds to Fig. 4(b).

\begin{tabular}{|c|c|}
\hline Physical parameter & Typical value \\
\hline \hline$h$ & $5 \times 10^{-6} \mathrm{~cm}(50 \mathrm{~nm})$ \\
\hline$\delta$ & $6.76 \times 10^{-8} \mathrm{~cm}(0.676 \mathrm{~nm})$ \\
\hline$\nu$ & $\left.0.5 \times 10^{14} \mathrm{~cm}^{-2}[19] 22\right]$ \\
\hline$\Omega$ & $2 \times 10^{-23} \mathrm{~cm}^{3}$ \\
\hline$T$ & $923 \mathrm{~K}$ \\
\hline$\beta$ & $5[14$ \\
\hline$\alpha_{i n t}$ & $16.3 \mathrm{erg} / \mathrm{cm}{ }^{2}[22]$ \\
\hline$\gamma_{A}, \gamma_{B}$ & $2.2 \times 10^{3} \mathrm{erg} / \mathrm{cm}^{2}([110] \mathrm{surface})[23$ \\
\hline$\epsilon$ & $1.2 \times 10^{-5} \mathrm{erg} / \mathrm{cm}[24]$ \\
\hline$\eta_{0}$ & $1.6 \times 10^{8} \mathrm{erg} / \mathrm{cm}{ }^{3}$ \\
\hline
\end{tabular}

TABLE I: Physical parameters. $\delta=4 R_{P d}, \Omega=4 \pi R_{P d}^{3} / 3, \gamma_{A}$ and $\gamma_{B}$ correspond to fcc CuPd alloy, with larger Pd atoms taken as deposited $B$ atoms in the model (i.e., $\mathrm{Pd}$ is the solute and $\mathrm{Cu}$ is the solvent). $R_{P d}=0.169 \mathrm{~nm}$ is the calculated radius of a $\operatorname{Pd}$ atom [25]. 'a

${ }^{a} \eta_{0}$ is estimated on the basis of LSA, which means that the stated dimensional value results in the dimensionless value $S=0.005$, with the latter value moderately affecting $\omega$ (Fig. 4) and not resulting in drastic nonlinear effects, such as phase separation solely due to the action of the compositional stress, see Fig. 6. (b).

(a)

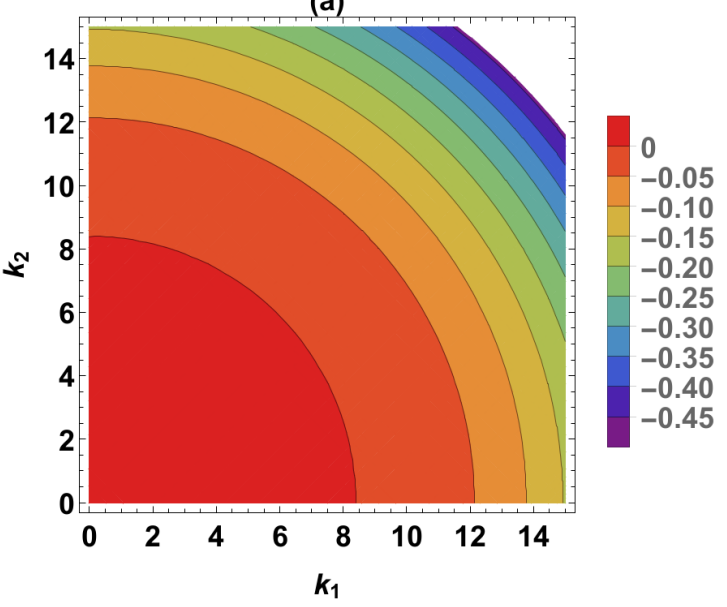

(b)

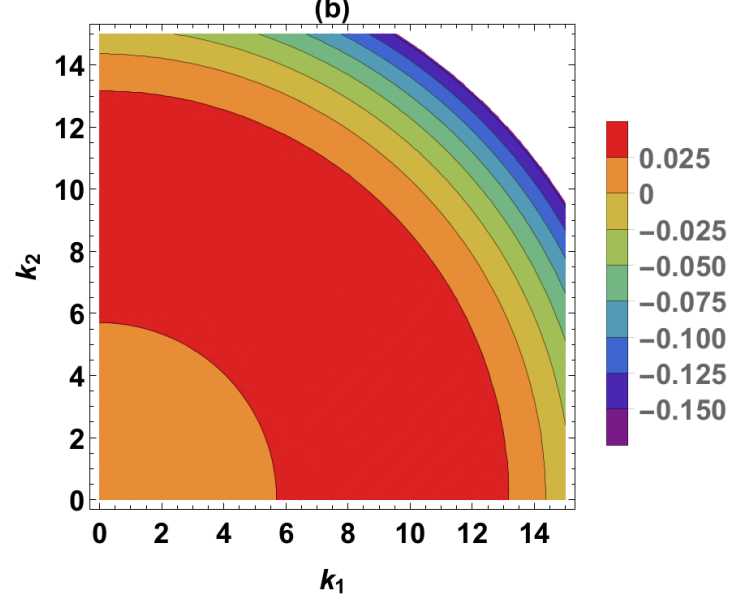

FIG. 4: The perturbation growth rate $\omega$ from LSA in the case of isotropy, i.e. $\beta=0$ and $\Lambda_{B}=1$, and $C_{B 0}=0.3$. (a): $\eta_{0}=0$ ( $S=0$, spinodal instability), (b): $\eta_{0}=1.6 \times 10^{8} \mathrm{erg} / \mathrm{cm}^{3}(S=0.005$, spinodal instability and compositional stress instability). 


\section{COMPUTATIONS OF THE COMPOSITION PATTERNS}

In this section we present the results of computations with Eq. (7). In all computations we use the initial condition $C_{B}(x, y, 0)=0.3+\xi(x, y)$, see Fig. 5(a), where $\xi(x, y)$ is the random deviation from the mean value 0.3 with the maximum amplitude 0.08 at a point $(x, y)$. Also, unless otherwise stated, we take the crystallographic anisotropy strength $\beta=5$ [14, and allow simultaneous spinodal and compositional stress instability, see Fig. (4) (b). Such choice enables comparison of the surface composition evolution for various values of the dimensionless parameters $\Lambda_{B}, S$, $\phi^{(x)}$, and $m$. Note that for all Figures, except Fig. 10 we chose $m=1$, i.e. [110] surface, and for all Figures, except Fig. 9 we use $\phi^{(x)}=30^{\circ}$. This angle is the convenient common value in the domains of the anisotropy functions for all three surface orientations.

Figures 5 and 6 show the evolution of the surface composition at zero diffusional anisotropy $\left(\Lambda_{B}=1\right)$. In Fig. 5 the evolution is computed until the time $t=500$. At this late stage $C_{B}$-rich domains show significant coarsening. The domains are starting to form around $t=70$ and up to around $t=200$ the pattern does not have a distinct preferred orientation. At later times the effect of the crystallographic anisotropy can be seen. The pattern is rotated clockwise by $45^{\circ}$ angle to the $y$-axis and slowly coarsens, so that the area fraction of large $C_{B}$ phase, $C_{B}>0.6$, increases (71\% in Fig. 5(c), $74 \%$ in Fig. 5(d), and 78\% in Fig. 5(e)). By pattern rotation angle we understand the prevailing inclination angle, to the $y$-axis of the plot, of the axes of the large- $C_{B}$ domains (colored red). A few of these axes are marked in each panel. Note that the phase-separated composition states, $C_{B} \approx 0.1$ (purple) and $C_{B} \approx 0.7$ (red) are stable in the figures, that is, the lower and the upper bounds on $C_{B}$ have been reached.
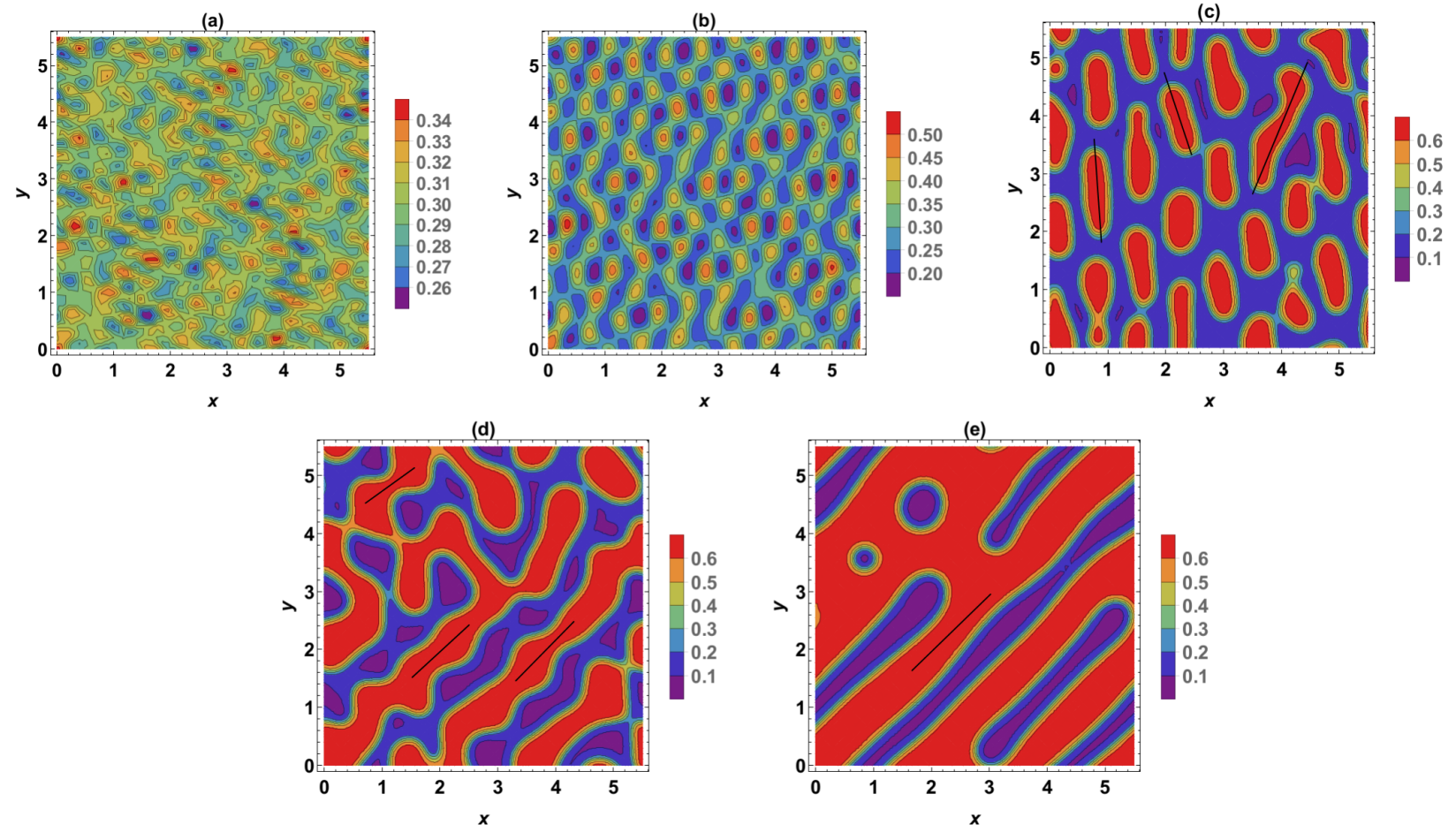

FIG. 5: (a): The example of the initial condition $C_{B}(x, y, 0),(\mathrm{b}): C_{B}(x, y, 10),(\mathrm{c}): C_{B}(x, y, 150),(\mathrm{d}): C_{B}(x, y, 350),(\mathrm{e})$ : $C_{B}(x, y, 500) . \Lambda_{B}=1, S=0.005, \phi^{(x)}=30^{\circ}, m=1$. The final dimensionless time $t=500$ corresponds to the physical time of $3 \mathrm{~min}$, assuming the typical surface diffusivity value $D_{B, \min }^{(x x)}=10^{-9} \mathrm{~cm}^{2} / \mathrm{s}$ at $T=923 \mathrm{~K}[26$.

In Fig. 6 the comparison is made of the patterns that resulted, at the fixed final time $t=250$, in the case of the parameters in Fig. 5 and the "reduced" situations, i.e. (i) absent the spinodal instability and (ii) absent the crystallographic anisotropy (since the diffusional anisotropy is already switched off, this means isotropy). When the spinodal instability is switched off (Fig. 6(b)) the phase separation is inhibited, meaning that the chosen level of the compositional stress alone is insufficient to cause phase separation. However, even on the nearly uniform concentration background, the orientation effect of the crystallographic anisotropy still can be clearly seen. When the crystallographic anisotropy is switched off (Fig. 6(c)) the domains are not preferentially oriented due to isotropy and they coarsen more slowly (71\% area fraction of large $C_{B}$ in Fig. 6(a) vs. $67 \%$ in Fig. 6(c)). The red domains are around $40 \mathrm{~nm}$ across in Fig. 6(a) and $30 \mathrm{~nm}$ across in Fig. 6)(c). 

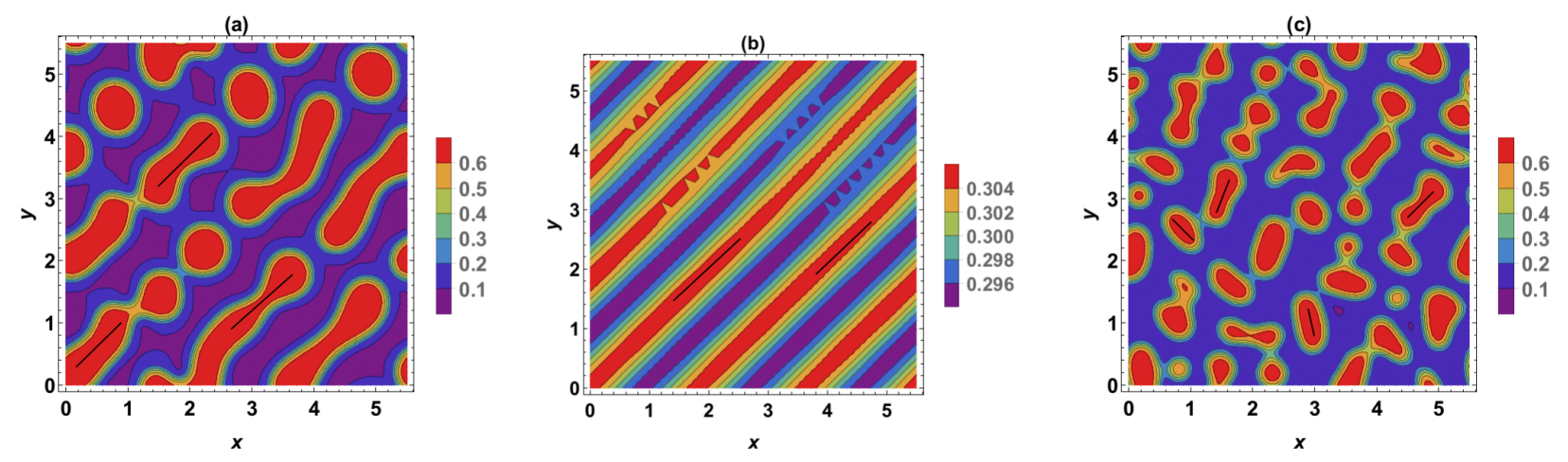

FIG. 6: (a): $C_{B}(x, y, 250) . \quad \Lambda_{B}=1, S=0.005, \phi^{(x)}=30^{\circ}, m=1$. Panel (b) shows $C_{B}(x, y, 250)$ computed with the same parameters as the panel (a), except $H=1.935$. In this case the free energy $\gamma$ is convex and the surface composition is thermodynamically stable. Panel (c) shows $C_{B}(x, y, 250)$ computed with the same parameters as the panel (a), except $\beta=0$. This is the isotropic case. 6.

In the following figures, for better comparisons, we plot the patterns at the same fixed final time $t=250$ as in Fig.

Variation of the solute expansion parameter $S$ in Fig. 7 has a strong impact on the pattern. As $S$ increases, so does the compositional stress in the surface layer, resulting in the sharply increasing area fraction of large $C_{B}$ phase. Also the circular shapes of $C_{B}$-rich domains at small $S$ are replaced by a worm-like shapes at moderate $S$ and by a "zipped" stripe shapes at large $S$. At later times (the snapshots not shown) coarsening results in the merging of the circular domains seen in Fig. 7(a) into a few dumbbell-shaped domains, which next merge to form zipped stripes tilted at $45^{\circ}$ angle to the $y$-axis, see Figures 7(c) and 5(e) for examples of this pattern; the worm-like domains seen in Fig. 7(b) also coarsen into the zipped stripes with the same orientation. Interestingly, large compositional stress eliminates the $45^{\circ}$ pattern rotation effect of the crystallographic anisotropy, as seen in Fig. 7(c).
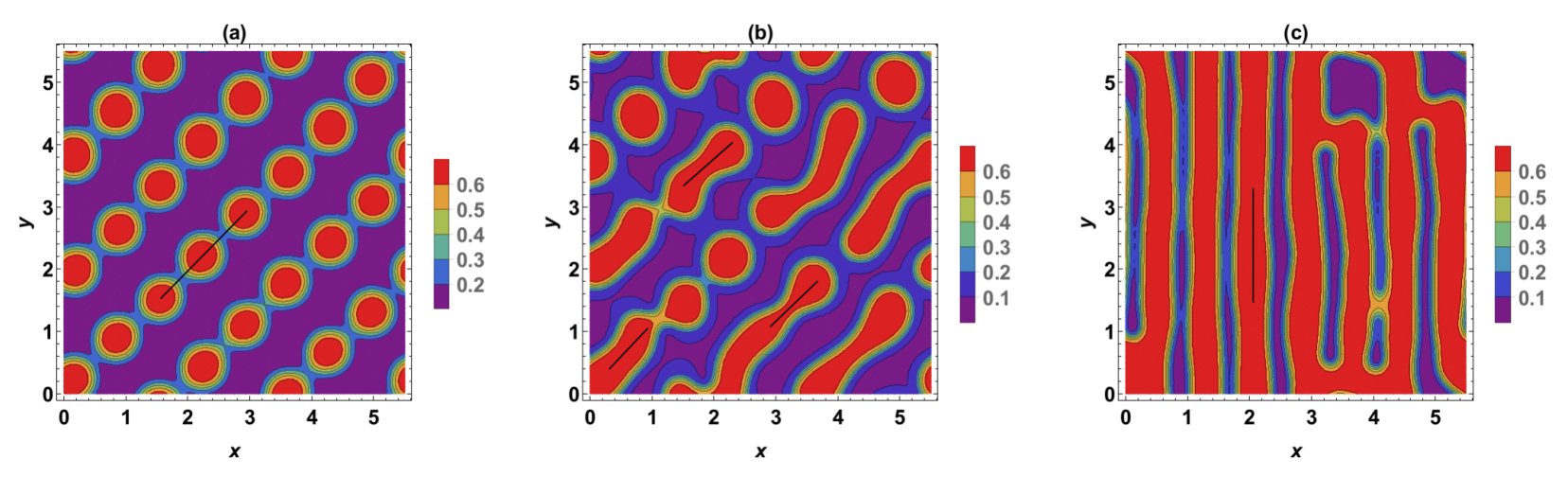

FIG. 7: (a), (b), (c): $C_{B}(x, y, 250)$ at $S=0.002,0.005,0.008$, respectively. $\Lambda_{B}=1, \phi^{(x)}=30^{\circ}, m=1$. The middle panel is the copy of Fig. 6 (a).

Fig. 8 shows the effect of the diffusional anisotropy $\Lambda_{B}$ coupled to the "background" crystallographic anisotropy. We observe that the pattern rotates counter-clockwise as value of $\Lambda_{B}$ is decreased from one, or it rotates clockwise as $\Lambda_{B}$ is increased from one. Increasing $\Lambda_{B}$ also has the effect of speeding up the coarsening. The diffusional anisotropy can be rationalized as follows. Without crystallographic anisotropy $(\beta=0)$ and at $\Lambda_{B}=1$ (i.e., when the diffusion strengths in the $x$ and $y$ direction are the same, $\left.D_{B, \min }^{(y y)}=D_{B, \min }^{(x x)}\right), \nabla_{\Lambda B}=\nabla$ and the spatial rates of change in the $x$ and $y$ directions are given by $\partial / \partial x$ and $\partial / \partial y$, thus the spatial variations in the pattern are similar in both directions. At $\Lambda_{B}=0.1$, the rate of change in the $y$-direction is roughly 10 times smaller than in the $x$-direction, thus the pattern looks more uniform in the $y$-direction than in the $x$-direction, i.e. the changes in the form of the alternation of small and large $C_{B}$ occur primarily in the $x$-direction. The overall impact is the pattern that consists of a worm-like bands of alternating large/small $C_{B}$ content (red/purple colored) running in the $y$ direction. At $\Lambda_{B}=10$ the situation is reversed. Close examination of the anisotropic gradient $\nabla_{\Lambda B}$ shows that the primary effect of the crystallographic anisotropy $\beta>0$ is to amplify the described effects of the diffusional anisotropy.

Fig. 9 shows that for [110] surface, the effect of the misorientation angle $\phi_{x}$ is to rotate the pattern by the angle $\phi_{x}+15^{\circ}$ clockwise from the $y$-axis. For $m=2$ case, i.e. [100] surface, there is no noticeable effect of the misorientation 
angle - the clockwise rotation from the $y$-axis is $30^{\circ}$ for all admissible misorientation angles. For $m=3$ case, i.e. [111] surface, the pattern can be mapped from the [110] pattern due to symmetry of the anisotropy functions, see Sec. II.
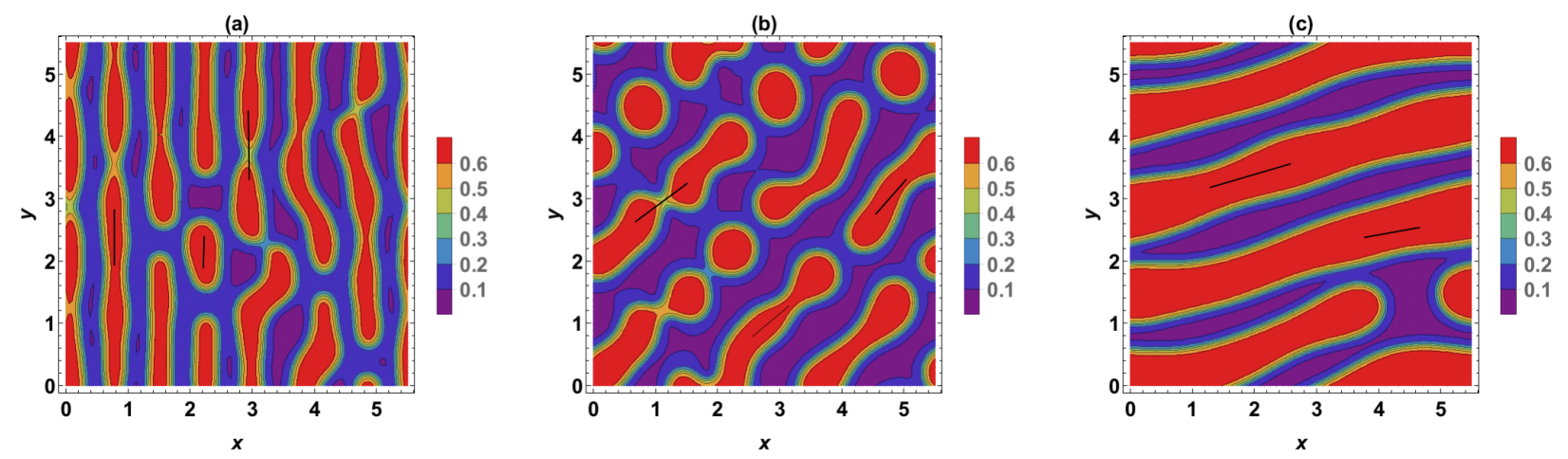

FIG. 8: (a), (b), (c): $C_{B}(x, y, 250)$ at $\Lambda_{B}=0.1,1,10$, respectively. $S=0.005, \phi^{(x)}=30^{\circ}, m=1$. The middle panel is the copy of Fig. 6(a).
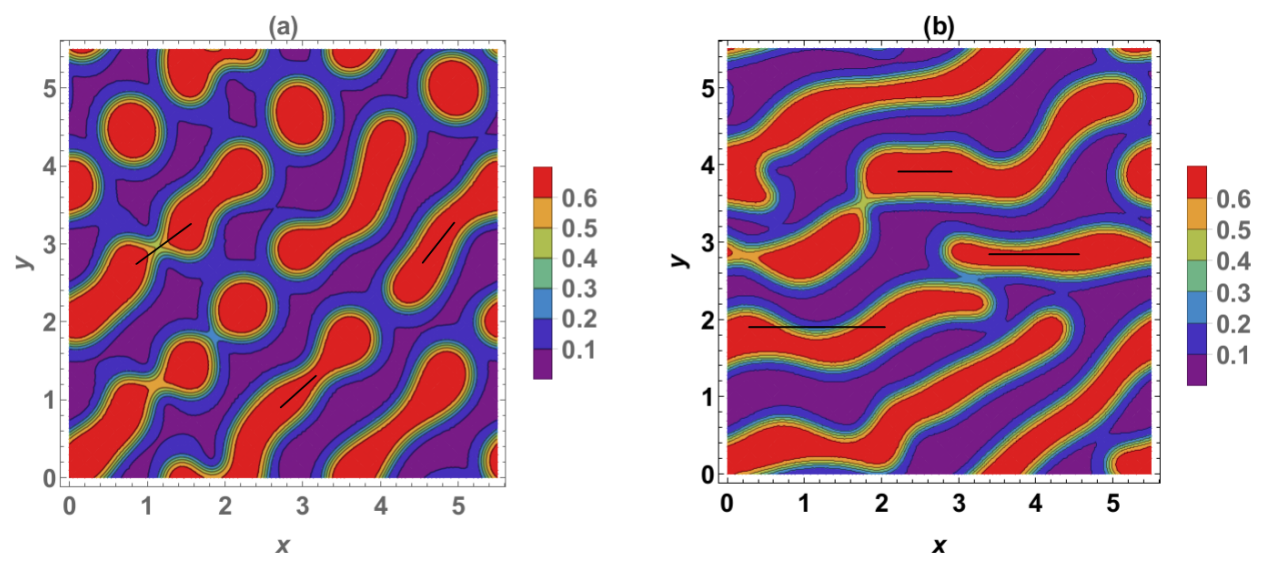

FIG. 9: (a), (b): $C_{B}(x, y, 250)$ at $\phi^{(x)}=30^{\circ}$ and $75^{\circ}$, respectively. $\Lambda_{B}=1, S=0.005, m=1$. The panel (a) is the copy of Fig. 8(b).

The last figure, Fig. 10, is the comparison of the composition patterns on three low-index surfaces, $[110](m=1)$, [100] $(m=2)$, and [111] $(m=3)$. We can notice the $45^{\circ}$ rotation angle for [110] surface, vs. the $30^{\circ}$ rotation angle for [100] surface, vs. $90^{\circ}$ angle for [111] surface. On the latter surface coarsening is faster than on [110] and [100] surfaces.
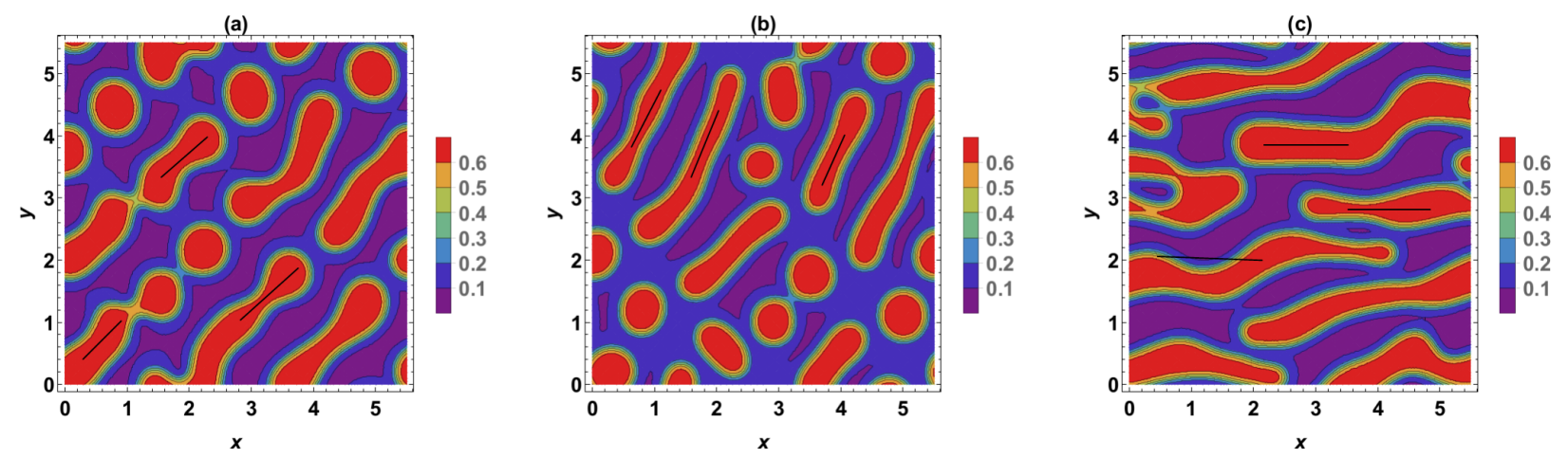

FIG. 10: (a), (b), (c): $C_{B}(x, y, 250)$ at $m=1,2,3$, respectively. $\Lambda_{B}=1, S=0.005, \phi^{(x)}=30^{\circ}$. The left panel is the copy of Fig. 6(a).

In Table II we summarize the computed orientations of the patterns for three low-index surfaces. We chose four equispaced values of $\phi^{(x)}$ in the admissible interval for each surface. As was pointed in Sec. III the pattern orientation 
angles for [110] and [111] surfaces coincide for such choice of $\phi^{(x)}$.

\begin{tabular}{|c|c|c|c|c|c|}
\hline Surface & $\phi^{(x)}=0$ & $\phi^{(x)}=b / 4$ & $\phi^{(x)}=b / 2$ & $\phi^{(x)}=3 b / 4$ & $\phi^{(x)}=b$ \\
\hline \hline$[110](m=1)$ & $0^{\circ}$ & $60^{\circ}$ & $45^{\circ}$ & $60^{\circ}$ & $90^{\circ}$ \\
\hline$[100](m=2)$ & $30^{\circ}$ & $30^{\circ}$ & $30^{\circ}$ & $30^{\circ}$ & $30^{\circ}$ \\
\hline$[111](m=3)$ & $0^{\circ}$ & $60^{\circ}$ & $45^{\circ}$ & $60^{\circ}$ & $90^{\circ}$ \\
\hline
\end{tabular}

TABLE II: The angle that the pattern axis makes with $y$-axis. $b=\pi / 2, \pi / 4$, and $\pi / 6$ for $m=1,2,3$, respectively. $\Lambda_{B}=1$, $S=0.005$.

To conclude, we note that the pattern orientation effect due to anisotropy once developed, remains constant throughout all stages of the coarsening and phase separation. For example, this can be seen in Fig. 5(e).

\section{CONCLUSIONS}

We analysed the basic continuum model for the in-plane composition patterning of an annealed surface alloy film, whereby the thin dynamic surface layer is the mixture of $A$ and $B$ atoms. A simultaneous action, in the surface layer, of a thermodynamic phase separation (spinodal decomposition), the compositional stress, and the kinetic effects which are due to anisotropy of the surface diffusivity is assumed.

By the systematic parametric computation, we found a robust phase separation of the surface layer into ordered composition patterns. Pattern in-plane orientation, the shape of the individual domains of the $B$-phase, its area fraction, and the pattern coarsening rate primarily depend on the parameters entering the anisotropic surface diffusivity tensor for $B$ atoms. This tensor, see Eq. (6), depends on the crystallographic orientation of the surface. The ratio of the amplitudes of the diagonal components of the tensor, $D_{B}^{(y y)} / D_{B}^{(x x)}$, as well as the value of the solute expansion coefficient (a measure of the compositional stress in the layer) make the largest impact on the pattern. The oriented meandering stripe pattern is typical in the late stages of the coarsening.

\section{APPENDIX}

The thermodynamic contribution $\frac{1-C_{B}}{\delta} \frac{\partial \gamma}{\partial C_{B}}$ in the surface chemical potential, Eq. 44, is stated by following the thermodynamically consistent derivation for bulk alloy in Ref. [19]. In that work, the form of the surface chemical potential is $\frac{1-C_{B}^{(b)}\left(z=z_{0}\right)}{\delta} \frac{\partial \gamma}{\partial C_{B}}$, where $C_{B}^{(b)}\left(z=z_{0}\right)$ is the bulk concentration at the surface (with $z_{0}$ marking the location of the surface layer). In our model of a surface alloy the bulk is the homogeneous A-phase, and we take $C_{B}^{(b)}\left(z=z_{0}\right)=C_{B}$. Most important, this treatment implies the correct incorporation of the pure element surface energy ratio $\Gamma$. As seen in Eq. 10), $\Gamma$ affects the linear stability. Moreover, taking $\Gamma \neq 1$ yields a concave $\gamma\left(C_{B}\right)$ curve, predicting total phase separation into $C_{B}=0$ and $C_{B}=1$ phases [20. This is confirmed by the simulation. Phase separation in this case is fast, allowing no time for anisotropic surface diffusion to form composition patterns. Inclusion of a phase-refining mechanism such as the surface stress 22 is expected to slow down the phase separation and to enable the final concentration states that are between zero and one, while the contribution of the pattern-orientation effect of the diffusion anisotropy is expected to stay constant.

\section{ACKNOWLEDGMENTS}

MK is grateful to Mark R. Bradley for useful discussions. VH's research was supported by the Perm Region Ministry of Science and Education (Grant C-26/798).

[1] F. Besenbacher, L.P. Nielsen, and P.T. Sprunger, "Surface alloying in heteroepitaxial metal-on-metal growth", in: The Chemical Physics of Solid Surfaces, Ed.: D.A. King and D.P. Woodruff, vol. 8, Elsevier (1997).

[2] G.L. Kellogg, "Surface alloying and de-alloying of Pb on single-crystal Cu surfaces", in: The Chemical Physics of Solid Surfaces, Ed.: D.P. Woodruff, vol. 10, Elsevier (2002). 
[3] H. Oughaddou, S. Sawaya, J. Goniakowski, B. Aufray, G. Le Lay, J. M. Gay, G. Tre'glia, P. Bibe’rian, N. Barrett, C. Guillot, A. Mayne, and G. Dujardin, "Ge/Ag(111) semiconductor-on-metal growth: Formation of an Ag2Ge surface alloy", Phys. Rev. B 62, 16653 (2000).

[4] C. Leandri, H. Oughaddou, B. Aufray, J.M. Gay, G. Le Lay, A. Ranguis, and Y. Garreau, "Growth of Si nanostructures on $\operatorname{Ag}(001) "$, Surf. Sci. 601, 262 (2007).

[5] H. Enriquez, A. Mayne, A. Kara, S. Vizzini, S. Roth, B. Lalmi, A.P. Seitsonen, B. Aufray, T. Greber, R. Belkhou, G. Dujardin, and H. Oughaddou, "Adsorption of silicon on Au(110): An ordered two dimensional surface alloy", Appl. Phys. Lett. 101, 021605 (2012).

[6] I.S. Choi, C.N. Whang, and C. Hwang, "Surface-induced phase separation in Pd-Ag alloy: the case opposite to surface alloying", J. Phys.: Condens. Matter 15, L415-L422 (2003).

[7] R. Plass, N.C. Bartelt, and G.L. Kellogg, "Dynamic observations of nanoscale self-assembly on solid surfaces", J. Phys.: Condens. Matter 14, 4227-4240 (2002).

[8] R. van Gastel, D. Kaminski, E. Vlieg, and B. Poelsema, "Surface alloying and anomalous diffusion of Bi on Cu(111)", Surf. Sci. 603, 3292-3296 (2009).

[9] Y. Girard, C. Chacon, G. de Abreu, J. Lagoute, V. Repain, and S. Rousset, "Growth of Bi on Cu(111): Alloying and dealloying transitions", Surf. Sci. 617, 118-123 (2013).

[10] J. Tersoff, "Surface-confined alloy formation in immiscible systems", Phys. Rev. Lett. 74, 434 (1995).

[11] J.W. Cahn and J. Hilliard, "Free energy of a nonuniform system. Interfacial free energy", J. Chem. Phys. 28, 258-267 (1958).

[12] J.W. Cahn, "On spinodal decomposition,", Acta Metall. 9, 795-801 (1961).

[13] G. Danker, O. Pierre-Louis, K. Kassner, and C. Misbah, "Peculiar effects of anisotropic diffusion on dynamics of vicinal surfaces", Phys. Rev. Lett. 93, 185504 (2004).

[14] L. Du and D. Maroudas, "Optimization of electrical treatment strategy for surface roughness reduction in conducting thin films", J. Appl. Phys. 124, 125302 (2018).

[15] S. Curiotto, P. Muller, A. El-Barraj, F. Cheynis, O. Pierre-Louis, and F. Leroy, "2D nanostructure motion on anisotropic surfaces controlled by electromigration", Appl. Surf. Sci. 469, 463-470 (2019).

[16] L. Du and D. Maroudas, "Theory of multiple quantum dot formation in strained-layer heteroepitaxy", Appl. Phys. Lett. 109, 023103 (2016).

[17] J. Renedo, R. Cuerno, M. Castro, and J. Munoz-Garcia, "Symmetry of surface nanopatterns induced by ion-beam sputtering: Role of anisotropic surface diffusion", Phys. Rev. B 93, 155424 (2016).

[18] M. Khenner, "Electromigration-guided composition patterns in thin alloy films: a computational study", Surf. Sci. 698, $121611(2020)$.

[19] Q. Zhang, P.W. Voorhees, and S.H. Davis, "Mechanisms of surface alloy segregation on faceted core-shell nanowire growth", J. Mech. Phys. Solids 100, 21-44 (2017).

[20] A.V. Ruban, H.L. Skriver, and J.K. Norskov, "Local equilibrium properties of metallic surface alloys", in: The Chemical Physics of Solid Surfaces, Ed.: D.P. Woodruff, vol. 10, Elsevier (2002).

[21] B.J. Spencer, P.W. Voorhees, and J. Tersoff, "Morphological instability theory for strained alloy film growth: The effect of compositional stresses and species-dependent surface mobilities on ripple formation during epitaxial film deposition", Phys. Rev. B 64, 235318 (2001).

[22] W. Lu and D. Kim, "Engineering nanophase self-assembly with elastic field", Acta Mater. 53, 3689 (2005).

[23] L. Vitos, A.V. Ruban, H.L. Skriver, and J. Kolla, "The surface energy of metals", Surf. Sci. 411, 186-202 (1998).

[24] J.J. Hoyt, "Molecular dynamics study of equilibrium concentration profiles and the gradient energy coefficient in $\mathrm{Cu}-\mathrm{Pb}$ nanodroplets", Phys. Rev. B 76, 094102 (2007).

[25] E. Clementi, D.L. Raimondi, and W.P. Reinhardt, "Atomic Screening Constants from SCF Functions. II. Atoms with 37 to 86 Electrons", J. Chem. Phys. 47, 1300 (1967).

[26] D. Amram, L. Klinger, and E. Rabkin, "Anisotropic hole growth during solid-state dewetting of single-crystal Au-Fe thin films", Acta Mater. 60, 3047-3056 (2012).

[27] U. Bardi, "The atomic structure of alloy surfaces and surface alloys", Rep. Prog. Phys. 57, 939 (1994).

[28] M.A. Vasiliev, "Surface effects of ordering in binary alloys", J. Phys. D: Appl. Phys. 30, 3037 (1997).

[29] For material B deposited on A, if B has lower surface energy $\left(\gamma_{B}<\gamma_{A}\right)$, then B tends to be confined to the surface layer; if $\gamma_{B}>\gamma_{A}$, then B tends to be dissolved in the bulk. CuPd fcc surface alloy ( $\mathrm{Cu}$ : A, Pd: B), chosen as the example system in section III, is neutral in terms of such surface energy effects, since $\gamma_{A} \approx \gamma_{B}$ for all major crystallographic surface orientations 23$]$.

[30] Note, when discussing the atomic content of the alloy surfaces, one has to clearly separate the cases of a surface of a bulk alloy (i.e., the "true" alloy surface), and the surface alloy. In reference to CuPd system, the former case was studied experimentally for the equilibrated (110) surface of a single-crystal $\mathrm{Cu}_{85} \mathrm{Pd}_{15}$ alloy [27, 28]. Two possible surface terminations were found: (i) a top pure $\mathrm{Cu}$ layer and a mixed second layer, and (ii) a topmost and second layer both mixed, with the first situation occurring more frequently. The latter case, which is relevant to this paper, was studied for (100) $\mathrm{CuPd}$ surface, showing that the topmost layer (with practically no buckling) is a mixed CuPd phase, and the second and subsequent layers are pure $\mathrm{Cu}$. Cu-based multilayer surface alloys (such that several top layers of $\mathrm{Cu}$ substrate are mixed with deposited atoms) were also prepared. The list of such surface alloys includes $\mathrm{CuAu}(100), \mathrm{CuPt}(111), \mathrm{CuFe}(100)$, and $\mathrm{CuSm}(100)$ [27. Our 2D model is concerned with the compositional variations in the surface plane (xy), which are averaged over the surface layer thickness, thus it does not resolve the composition profile across the surface layer. 